\title{
EFECTO FOTOPROTECTOR DE Macrocystis pyrifera FRENTE A BLOQUEADORES SOLARES EN LA PREVENCIÓN DE LESIONES DE PIEL
}

\section{Photoprotective effect of Macrocystis pyrifera compared to sunscreens in prevention of skin lesions}

Américo J. Castro ${ }^{1}$, Mario Carhuapoma ${ }^{1}$, Norma J. Ramos ${ }^{1}$, José R. Juárez ${ }^{1}$, Luis M. Felix ${ }^{1}$, José F. Jáuregui ${ }^{1}$, Julio P. López ${ }^{1}$, Luis A. Inostroza ${ }^{1}$, Omar Santa María ${ }^{1}$, Paul I. Gutierrez ${ }^{1}$, Sixto A. Gonzales ${ }^{1}$, Ada L. Javier ${ }^{1}$, Jackeline Escudero ${ }^{1}$, Arnaldo J. Navarro $^{1}$, Susan A. Huamán, Merrit B. Machaca

${ }^{1}$ Instituto de Investigación en Ciencias Farmacéuticas y Recursos Naturales "Juan de Dios Guevara”, Facultad de Farmacia y Bioquímica, Universidad Nacional Mayor de San Marcos

\section{RESUMEN}

El estudio tuvo como objetivo comparar el efecto fotoprotector in vivo de una crema elaborada con el extracto hidroalcohólico de la macroalga parda Macrocystis pyrifera (Linnapus) Agardh frente a bloqueadores solares comerciales en la prevención de lesiones de piel. La muestra biológica fue colectada en el zócalo continental de la playa Yanyarina de San Juan de Marcona de la región Ica. El extracto hidroalcohólico (etanol-agua 1:1) se obtuvo por proceso de maceración a partir de $300 \mathrm{~g}$ de alga. Con el extracto se formuló y diseño el bloqueador dermocosmético empleando concentraciones 5 , 10 y $15 \%$, respectivamente, con una mezcla de alcoholes alifáticos sólidos, conformados principalmente por alcohol cetílico $\left(\mathrm{C}_{16} \mathrm{H}_{3} \mathrm{O}\right)$ yalcohol estearílico $\left(\mathrm{C}_{18} \mathrm{H}_{39} \mathrm{O}\right)$ $50 / 70 \%$. Se emplearon 35 ratones albinos adultos machos de la especie Mus musculus cepa Bald $C_{53}$ de peso promedio $30 g$. El potencial fotoprotector in vivo se evaluó a través de la aplicación tópica en la piel de los ratones, en el tercio anterior dorsal del lomo. Los animales fueron distribuidos en siete grupos de cinco. Al grupo 1 (blanco) no se le aplicó crema ni irradiación UV-B 320 ym; al grupo 2 (control) no se le aplicó crema y se le irradió con luz UV-B; los grupos 3 y 4 fueron tratados con bloqueadores solares comerciales y fueron irradiados con luz UV-B; a los grupos 5, 6 y 7 (intervención) se les aplicó el producto elaborado y se le irradió con luz UV-B. El efecto fotoprotector se evidenció en el análisis macroscópico y microscópico de la piel. En el análisis microscópico, el grupo 1 presento piel con estructura conservada; los grupos 2, 3 y 4 presentaron estructura alterada; mientras que el grupo 5 presentó alteración leve, el grupo 6, estructura aparentemente normal y el grupo 7 estructura normal. Se concluye que el extracto hidroalcohólico ejerce efecto fotoprotector in vivo ante la irradiación UV-B en las concentraciones trabajadas, lográndose que, a medida que se va aumentando la concentración del extracto, la estructura de la piel se ve menos alterada presentando la dermis y epidermis, la capa cornea laminary basal aparentemente normales.

Palabras clave: Macrocystis pyrifera, Mus musculus, fotoprotector, bloqueador solar.

\section{SUMMARY}

Objective of the study was to compare the sunscreen effect in vivo of a cream made with the hydroalcoholic extract of the brown seaweed Macrocystis pyrifera (Linnapus) Agardh, with comercial sunscreens in skin lesions prevention. The biological sample was collected in the continental socket from Yanyarina beach, San Juan de Marcona, Ica region. Hydroalcoholic extract (ethanol 1:1) was obtained for maceration process since 300 g seaweed. With the extract was formulated and design the blocker dermocosmetic using concentrations of 5,10 , and $15 \%$, respectively, with a mixture of solids aliphatic alcohols, consisting mainly of Cetyl alcohol $\left(\mathrm{C}_{16} \mathrm{H}_{3} \mathrm{O}\right)$ and Stearyl alcohol $\left(\mathrm{C}_{18} \mathrm{H}_{39} \mathrm{O}\right){ }_{50} \mathrm{O} / 70 \%$. Were used albino mice, mus musculus specie, bald c53 strain, adult males, with average weight $30 \mathrm{~g}$. Photoprotective potential in vivo was tested through topical application on mice skin, in the dorsal anterior third of loin. The animals were distributed into seven groups of five. Group 1 (white) was not applied cream or irradiation UV-B 320 ym; Group 2 (control) was not applied cream and was irradiated with UV-B light; groups 3 and 4 were treated with commercial sunscreens and irradiated with UV-B light; groups 5, 6 and 7 (intervention) were applied the processed product and were irradiated them with UV-B. The sunscreen effect was evident in macroscopic and microscopic analysis of skin. In the microscopic analysis, group 1 presented skin with preserved structure; groups 2, 3 and 4; had altered structure; while group 5 presented slight alteration, group 6, structure seemingly normal and group 7 structure normal. It was concluded that the hydroalcoholic extract exerts sunscreen effect in vivo against UV-B irradiation on worked concentrations; achieving more protection when is used a major extract concentration, this was evidenced because the skin structure looks less altered with dermis and epidermis, layer corneal lamellar and basal apparently normal.

Keywords: Macrocystis pyrifera, Mus musculus, fotoprotector, sunscreen.

\section{INTRODUCCIÓN}

M acrocystis pyrifera (Linnapus) Agardh es una alga parda gigante que se desarrolla en forma silvestre en el litoral peruano y posee en su composición química compuestos que actúan evitando el envejecimiento de la piel. Estos compuestos exhiben un abanico de cualidades beneficiosas aplicables para la salud, por lo que se les considera en los campos cosmético y alimenticio, entre otros ${ }^{(1)}$. El color pardo se debe a la presencia de sustancias xantofilas y flavoxantina, clorofila y otros pigmentos. Son algas eucarióticas, pluricelulares y morfológicamente muy diversas, de crecimiento rápido y de gran tamaño, pudiendo alcanzar hasta $30 \mathrm{~m}$ de largo ${ }^{(2)}$.

La piel es un tegumento externo que cumple función protectora, hace intercambio acuoso y térmico con el medio externo y sintetiza vitamina $\mathrm{D}$, es por ello que su mantenimiento es importante para el organismo; asimismo, sufre un proceso de envejecimiento, intrínseco y extrínseco, siendo dañina para ella la radiación ultravioleta (UV) ${ }^{(3)}$. Esta radiación afecta las fibras colágenas y elásticas, los fibroblastos, las células inmunes cutáneas, la microvasculatura y diferentes tipos celulares del estrato epidérmico ${ }^{(4)}$. Son muchos los investigadores que buscan modelos biológicos que permitan estudiar el envejecimiento de la piel y a la vez conocer formulaciones de acción tópica, a base de recursos naturales, que contrarresten o detengan las alteraciones de este órgano ${ }^{(5)}$. Se han evaluado diversos compuestos de origen marino como agentes protectores capaces de contrarestar la acción de la radiación ultravioleta ${ }^{(6)}$. 
El objetivo de esta investigación fue comparar el efecto fotoprotector in vivo de una crema dermocosmética con extracto hidroalcohólico del alga Macrocystis pyrifera (Linnapus) Agardh, preparada a concentraciones de 5, 10 y $15 \%$, frente a bloqueadores solares comerciales en la prevención de lesiones de piel.

\section{MATERIALES Y MÉTODOS}

La investigación es experimental y el diseño es descriptivo, analítico, prospectivo y longitudinal.

\section{Colecta del material biológico}

Las algas fueron colectadas en el zócalo continental de la playa Yanyarina de Marcona, en la región Ica, y su clasificación taxonómica se realizó en el Museo de Historia Natural de la Universidad Nacional Mayor de San Marcos, según el sistema de clasificación de Güiry, M.D.\& Güiry G. M. 2013:

División: Phaeophyta

Clase : Pheophyceace

Orden : Laminariales

Género : Macrocystis

Especie: Macrocystis pyrifera (Linnapus) Agardh

\section{Obtención del extracto hidroalcohólico}

Las algas fueron tratadas con agua de mar y posteriormente con agua potable, secadas a $40^{\circ} \mathrm{C}$, molidas y tamizadas. El extracto hidroalcohólico (etanol-agua 1:1) se obtuvo de $500 \mathrm{~g}$ de alga por proceso de maceración y posterior concentración a presión reducida en un evaporador rotatorio.

\section{Formulación de la crema}

Con el extracto se formuló y diseño el bloqueador dermocosmético natural, empleando concentraciones de $5,10,15 \%$ del extracto, en una mezcla de alcoholes alifáticos sólidos conformados principalmente por el alcohol cetílico $\left(\mathrm{C}_{16} \mathrm{H}_{3} \mathrm{O}\right)$ y alcohol estearílico $\left(\mathrm{C}_{18} \mathrm{H}_{39} \mathrm{O}\right){ }_{50 / 70 \%}$.

\section{Efecto fotoprotector del extracto hidroalcohólico}

Se determinó sobre la piel de ratones irradiados con luz UV-B cada 12 horas durante siete días.

\section{Animales de experimentación}

El estudio se realizó en 35 ratones albinos adultos machos de la especie Mus musculus cepa Bald $\mathrm{C}_{53}$, de un peso promedio de $30 \mathrm{~g}$, que fueron distribuidos en siete grupos de cinco: grupo 1 blanco, grupo 2 control, grupos 3 y 4 (tratados con bloqueadores solares comerciales), y grupos 5, 6 y 7 (tratados con crema al 5, 10 y $15 \%$ ).

\section{RESULTADOS}

El efecto fotoprotector de la crema con el extracto hidroalcohólico de Macrocystis pyrifera (Linnapus) Agardh, se presenta en la tabla 1.

Los resultados de los cortes histológicos de los grupos estudiados, se presentan en las figuras 1 a 7 .

\section{DISCUSIÓN}

La radiación solar causa daños sobre la piel a través de la radiación ultravioleta (UV-B-290/3201m), asimismo
Tabla 1. Efecto fotoprotector de la crema sobre la piel de ratones irradiados con luz UV-B.

\begin{tabular}{|c|c|c|}
\hline \multirow[b]{2}{*}{ Grupos } & \multicolumn{2}{|c|}{ Después de siete días } \\
\hline & $\begin{array}{c}\text { Análisis } \\
\text { macroscópico }\end{array}$ & $\begin{array}{l}\text { Análisis } \\
\text { microscópicos }\end{array}$ \\
\hline $\begin{array}{l}\text { Grupo } 1 \text { (blanco) } \\
\text { Depilados, no irradiados y } \\
\text { sin crema }\end{array}$ & $\begin{array}{l}\text { Piel con estructura } \\
\text { normal }\end{array}$ & $\begin{array}{l}\text { Piel con estructura } \\
\text { conservada. }\end{array}$ \\
\hline $\begin{array}{l}\text { Grupo } 2 \text { (control) } \\
\text { Depilados, irradiados y sin } \\
\text { crema }\end{array}$ & $\begin{array}{l}\text { Piel sensible al tacto } \\
\text { Dañada } \\
\text { Resecamiento } \\
\text { Arrugada } \\
\text { Enrojecimiento }\end{array}$ & $\begin{array}{l}\text { Piel con estructura } \\
\text { alterada }\end{array}$ \\
\hline $\begin{array}{l}\text { Grupo } 3 \text { (intervención) } \\
\text { Depilados, irradiados y con } \\
\text { crema "A" }\end{array}$ & $\begin{array}{l}\text { Piel muy enrojecida } \\
\text { Reseca } \\
\text { Arrugada }\end{array}$ & $\begin{array}{l}\text { Piel con estructura } \\
\text { alterada }\end{array}$ \\
\hline $\begin{array}{l}\text { Grupo } 4 \\
\text { (intervención) } \\
\text { Depilados, irradiados y con } \\
\text { crema "B" }\end{array}$ & $\begin{array}{l}\text { Piel muy enrojecida } \\
\text { Menor arrugamiento } \\
\text { Sequedad }\end{array}$ & $\begin{array}{l}\text { Piel con estructura } \\
\text { alterada }\end{array}$ \\
\hline $\begin{array}{l}\text { Grupo } 5 \\
\text { (intervención) } \\
\text { Depilados, irradiados y con } \\
\text { crema al } 5 \%\end{array}$ & $\begin{array}{l}\text { Piel seca } \\
\text { Leve eritema }\end{array}$ & $\begin{array}{l}\text { Leve alteración } \\
\text { estructural }\end{array}$ \\
\hline $\begin{array}{l}\text { Grupo } 6 \\
\text { (intervención) } \\
\text { Depilados, irradiados y con } \\
\text { crema al } 10 \%\end{array}$ & $\begin{array}{l}\text { Piel seca } \\
\text { Aparente eritema }\end{array}$ & $\begin{array}{l}\text { Estructura } \\
\text { aparentemente } \\
\text { normal }\end{array}$ \\
\hline $\begin{array}{l}\text { Grupo } 7 \\
\text { (intervención) } \\
\text { Depilados, irradiados y con } \\
\text { crema al } 15 \%\end{array}$ & $\begin{array}{l}\text { Piel levemente seca, } \\
\text { Sin eritema }\end{array}$ & Estructura normal \\
\hline
\end{tabular}

genera especies reactivas de oxígeno que provocan envejecimiento caracterizado por pigmentación, pérdida de elasticidad, arrugas y riesgo de cáncer a la piel ${ }^{(7)}$.

Se conoce que la radiación UV destruye totalmente la microvasculatura, generando alteraciones como congestión y hemorragia (8). En los fenómenos de envejecimiento de la piel, intervienen procesos de degradación de fibras colágenas y de las fibras elásticas (9). En estudios morfológicos realizados por diferentes autores, se ha demostrado que bajo la influencia de la luz UV se destruye el colágeno y aumenta el número de las fibras elásticas ${ }^{(10)}$.

La revisión bibliográfica demuestra que las macroalgas pardas producen gran variedad de metabolitos secundarios, encontrándose entre éstos polifenoles, polisacáridos, terpenos y otros, que ofrecen un amplio espectro de actividades biológicas, siendo una de ellas la actividad antiinflamatoria ${ }^{(11-13)}$.

Estudios realizados con extractos de algas marinas de las costas cubanas, permitieron evaluar su actividad antifotoenvejecimiento, encontrándose que las cremas preparadas con 25,50 y $100 \mathrm{mg} / \mathrm{mL}$ de extracto de algas y aplicadas sobre ratones machos después de irradiación UV, atenuaban las alteraciones histopatológicas causadas (14). Las algas pardas producen una amplia variedad de metabolitos secundarios, que incluyen sustancias de tipo polisacárido como son los fucanos, algunos de éstos sulfatadosy conactividad anticoagulante, antiinflamatoria y antitumoral $(15,16)$. En Macrocystis pyrifera (Linnapus) Agardh, también se ha estudiado presencia de actividad antiinflamatoria, analgésica y antioxidante ${ }^{(17)}$. Estudios realizados con gel de Vimang, con conocida actividad antifotoenvejecimiento, que incluyeron análisis 
histopatológico, concluyeron que las mejorías observadas macro y microscópicamente evidenciaban su acción fotoprotectora ${ }^{(18)}$.

La consideración ética en el tratamiento de los animales de experimentación se efectuó con rigor y calidad científica ${ }^{(19)}$. La formulación de la crema utilizada en este estudio y los excipientes utilizados se aplicaron de acuerdo a las referencias especializadas en esta área ${ }^{(20,21)}$.

\section{CONCLUSIÓN}

El extracto hidroalcohólico de la macroalga parda Macrocystis pyrifera (Linnapus) Agardh, presenta efecto fotoprotector in vivo en la forma farmacéutica de crema al 5, 10 y $15 \%$, respectivamente, para la prevención de lesiones en piel.

\section{REFERENCIAS BIBLIOGRÁFICAS}

1. Kinsella JE, Frankel E, German B, Kanner J. Possible mechanisms for the protective role of antioxidants in wine an plant foods. Food Technology. 1993; 47(4): 85-9.

2. Santelices B, Ojeda P. Effects of canopy removed on this understory algal community structure of coastal forests of Macrocystis pyrifera from southern South America. Marine Ecology Progress Series. 1989 (1984); 14: 165-73.

3. Ledo A. Clinical aspects of intrinsic aging and associated dermatosis. The procceding of the 18th World Congress of Dermatology. 1992: 823 .

4. Bernstein EF, Chen YQ, Kopp JB, Fisher L, Brown DB, Hahn PJ, et al. Long-term sun exposure alters the collagen of the papillary dermis. Comparison of sun-protected and photoaged skin by northern analysis, immunohistochemical staining, and confocal laser scanning microscopy. J Am Acad Dermatol. 1996; 34(2 Pt 1): 209-18.

5. Gilchrest BA. A review of skin ageing and its medical therapy. J Dermatol. 1966; 135(6): 867-75.

6. Dunlap WC, Chalker BE, Bandaranayake WM. Wu Won JJ. Nature's sunscreen from the Great Barrier Reef, Australia. Int J Cosmetic Sci. 1998; 20(1): 41-51.

7. Chancal D, Swarlata S. Herbal photoprotective formulation and their evaluation. Open Nat Prod J. 2009; 2: 71-6.

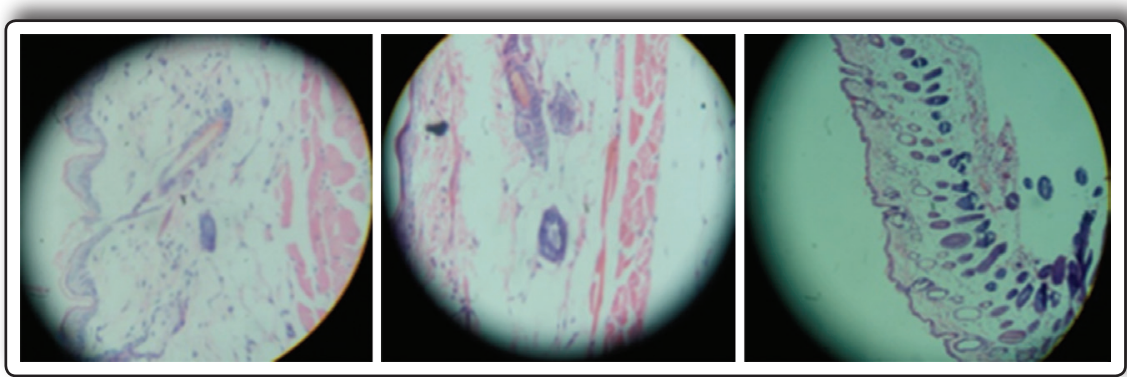

Figura 1. Blanco, ratones depilados, sin crema y sin irradiación (100X-400X). Estructura conservada: dermis, epidermis y capa muscular conservada, capa cornea laminar y filamentosa, tejido colágeno laxo, fibras colágenas regulares y presencia de folículos pilosos; macrófagos y células sebáceas normales.

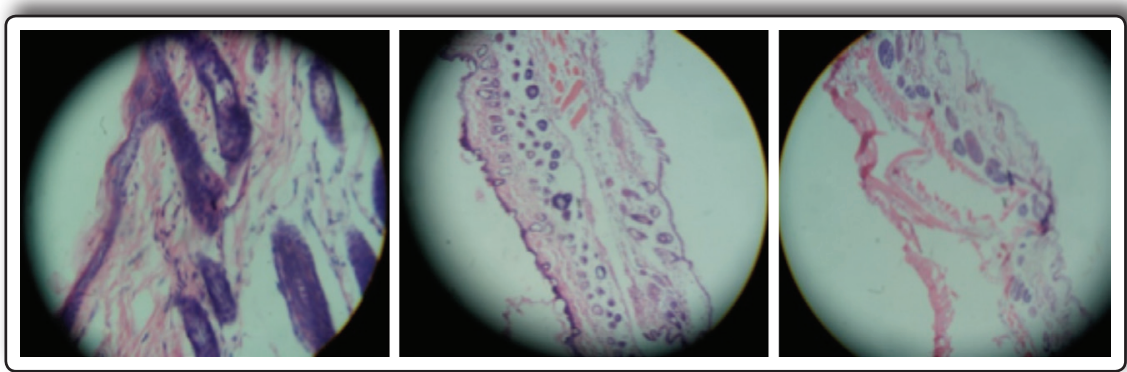

Figura 2. Control, depilado e irradiado (100X-400X). Estructura alterada: capa cornea laminar con alteraciones y coagulada, colágeno compacto, necrosis de folículos pilosos, capa muscular edematosa y necrosada, células epidérmicas sin núcleo y otras con restos nucleares, tejido conjuntivo laxo y edematoso, folículos pilosos con capa disminuida, elastina delgada y fibras musculares coaguladas, edema ampollado, capa cornea discontinua, epidermis adelgazada, dermis con ampollas y tejido muscular ralo.

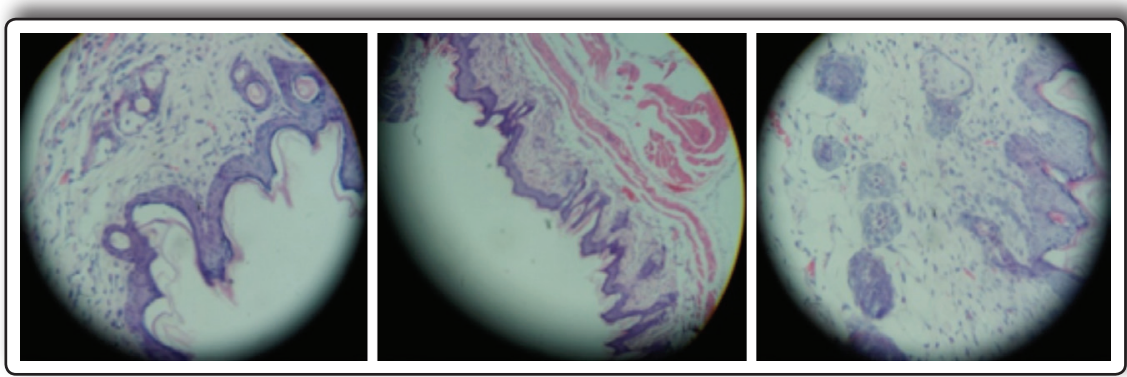

Figura 3. Grupo "A" Nonsun, depilados e irradiados (100X-400X). Estructura alterada: escasa capa cornea, membrana basal tenue, dermis reemplazada por tejido fibroso, fibroblastos laxo, congestión, tejido conjuntivo laxo y compacto, capa muscular gruesay discretamente edematosa, discretos folículos pilosos conservados.
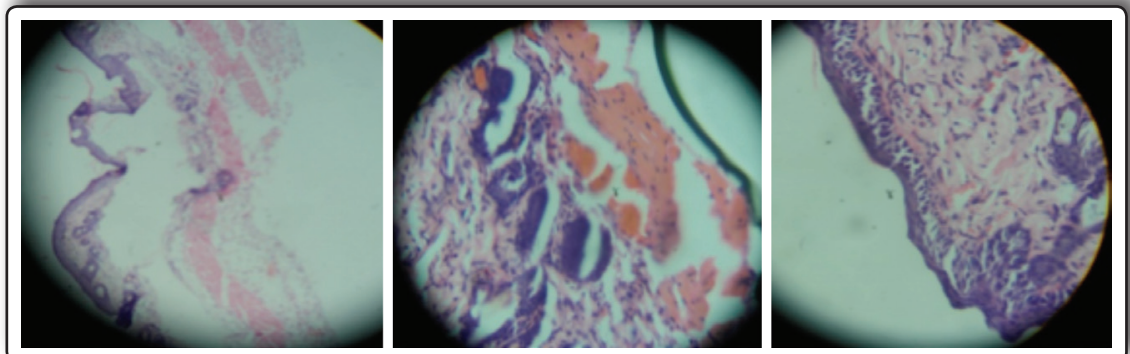

Figura 4. Grupo "B" Tecnoskin, depilados e irradiados (100X-400X). Estructura alterada: capa cornea ausente y compacta, células epidérmicas separadas, capa basal compacta y gruesa, presencia de colágenos con fibroblastos que envuelven a los folículos pilosos, tejido muscular en haces compacto, edematoso y alterado; células edematosas, tejido conjuntivo laxo con polinucleares y macrófagos; capa cornea separada de la epidermis, tejido conjuntivo laxo, infiltración de macrófagos, linfocitos y células de fibroblastos, capa basal edematosa, edema subepidérmico e intraepidérmico.
8. Beissert S, Granstein RD. UV induced cutaneous photodamage. Biochem Mol Biol. 1996; 8: 381-404.

9. Davis S, Lopjoch L, Kerr N, Fedesejeris R. Clothing as protection from UV radiation: which fabric is most 
effective? Int J Dermatol. 1997; 36(5): 374-9.

10. Hatarake A. Uchida Y, Mimura, Elias PM, Holleran WM. Intrinsically aged epidermis displays diminished UVB-induced alteration in barrier function associated with decreased proliferation. J Invest Dermatol. 1997; 108(3): 319-23.

11. Menéndez R, Fernández MD, García N. Las algas marinas como fuente de nuevos agentes antiinflamatorios. Revista electrónica de la Agencia de Medio Ambiente. Citado 24 noviembre 2014. 2010: 19. http://ama.redciencia.cu/ articulos/19.06.pdf.

12. García N, Frías AL, Cabrera $\mathrm{H}$, Menéndez $M$, Del valle $S$, Sierra Y, Suárez AM. Anti-inflamatory and antinociceptive activities of methanolic extract from red seaweed Dichotomaria obtusata, Brazilian Journal of Pharmaceutical Sciences, 2013; 49(81): 66-70.

13. Smit AJ. Medicinal and pharmaceutical uses of seaweed natural products: a review. J Appl Phycol. 2004; 16: 245.

14. Concepción A, Fernández M, Fernandez A, Cruz T. Evaluación de los extractos de algas marinas, con actividad antioxidante y reorganizadora de la fibra colágena. Rev Cubana Invest Biomed. 2001; 20 (1): 6-11.

15. Chang $H$, Jang $K H$, Lee $D$, et al. Monoglycerides from the Brown alga Sargassum sagamianum: Isolation, synthesis, and biological activity. Bioorg Med Chem Lett.
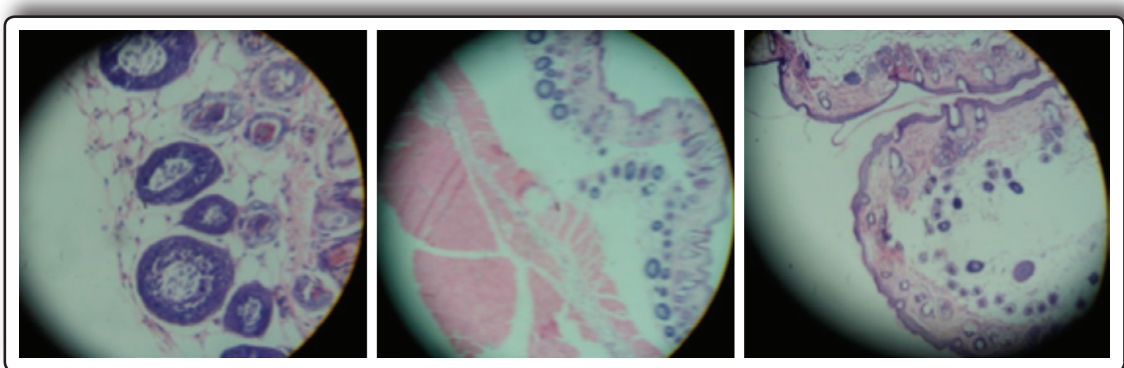

Figura 5. Grupo con extracto 5\%, depilados eirradiados (100X-400X). Leve alteración estructural: aumento de tejido compacto, discreto edema, capa cornea y epidermis conservados, epitelio normal, capa basal conservada, escasas infiltraciones, folículos pilosos normales, capa muscular gruesa con núcleos, edema en los tejidos (infiltración de líquidos), piel delgada sin alteraciones, tejido conjuntivo laxo casi normal, presencia de membrana basal, discreta alteración de folículos pilosos, tejido muscular subcutáneo normal, colágeno laxo y edematoso.

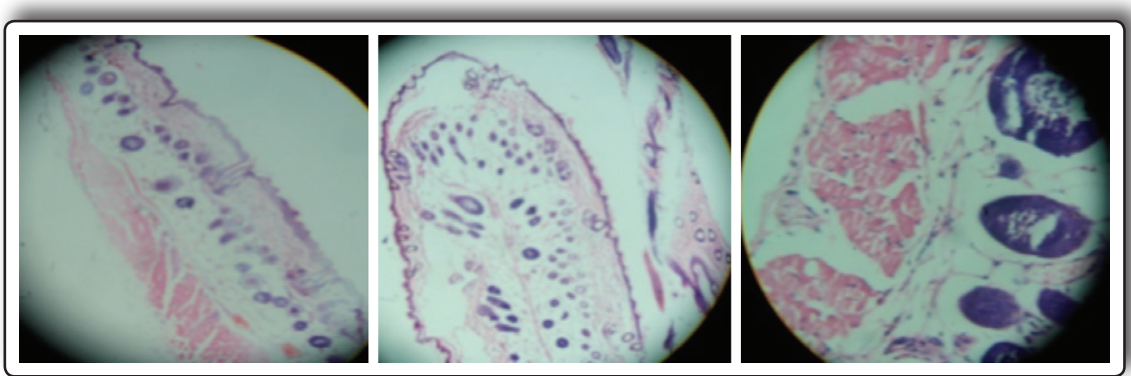

Figura 6. Grupo con extracto 10\%, depilados e irradiados (100X-400X). Estructura normal: aumento de colágeno con fibroblastos y macrófagos, epidermis normal, capa cornea laminar y basal normal, presencia de tejido celular y folículos pilosos sin alteraciones, buena irrigación y presencia de epitelio graso.

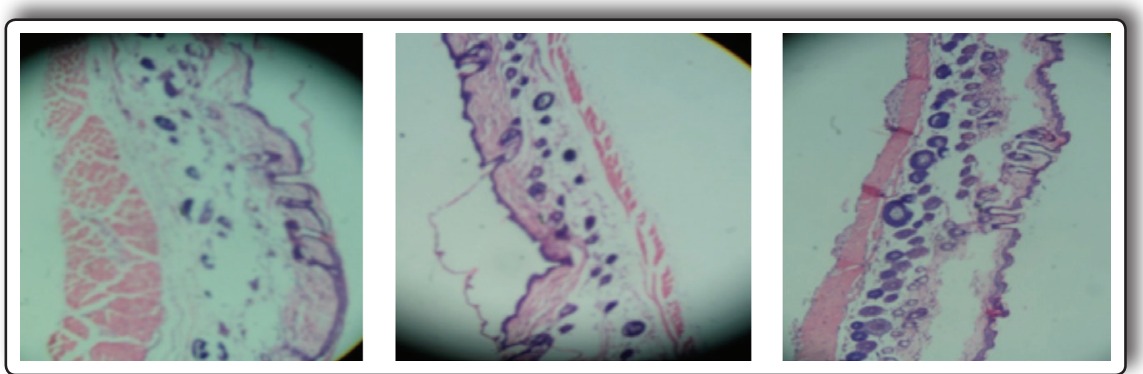

Figura 7. Grupo con extracto 15\%, depilados e irradiados (100X-400X). Estructura normal: capa cornea sin alteraciones, capa basal normal, presencia de folículos pilosos normal sin alteraciones, presencia de tejido muscular normal, glándulas sebáceas, tejido conjuntivo laxo, fibroblastos normales. 2008; 18 (12): 3589-92.

16. Queiroz KC, Medeiros VP, et al. Inhibition of reverse transcriptase activity of hiv by polysacharides of brown algae. Biomed pharmacother. 2008; 62(5): 303-7.

17. Ruth M, Galurdo C, et al. Propiedades antiinflamatorias, analgésicas y antioxidantes de las altas marinas de la Región Ica. Asociación Científica de Investigación Farmacéutica-ACIF. El Químico Farmacéutico. Revista del Colegio Químico Farmacéutico Departamental de Lima, $\mathrm{N}^{\circ} 1$ Año 1-julio 2012.

18. Sánchez I, González J, Zetina C, Casanova R. Análisis de la biodiversidad de algas marinas situadas entre Uaymitún y Chuburná, Mérida - Mexico. 2007; 11(1): 43-51.

19. Cardoso de Martínez CA, Mrad de Osorio A. Ética en Investigación con animales: Una actitud responsable y
Correspondencia:
$\begin{array}{ll}\text { Nombre: } & \text { Américo Jorge Castro Luna } \\ \text { Dirección: } & \text { Jr. Puno 1002, Lima 1 - Perú } \\ \text { Email: } & \text { CASLASHA3@hotmail.com }\end{array}$

respetuosa del investigador con rigor y calidad científica. Revista Latinoamérica de Bioética. 20o8; 8(2): 46-71.

2o. Hanbook of Pharmaceutical. Excipients. $5^{\text {ta }}$ ed. London. Chicago; 2005.

21. Farmacopea de los Estados Unidos de Norteamérica y Formulario Nacional (USP 31-NF269. Maryland. The United States Phamacopeial Convention. 2008.

Manuscrito recibido el: 28/09/2015

Aceptado para su publicación el: 02/11/2015 